\title{
Megaesôfago idiopático: relato de caso
}

\section{Idiopathic megaesophagus: a case report}

DOI: $10.46814 /$ lajdv3n4-074

Recebimento dos originais: 01/05/2021

Aceitação para publicação: 31/06/2021

\section{Artelho de Freitas Guimarães Júnior}

Formação acadêmica: médico generalista

Instituição de atuação atual: Hospital Municipal Universitário, Rio Verde - Goiás Endereço completo: av. Jerônimo Martins, s/n, Parque Bandeirante - Rio Verde, Goiás E-mail: artelhojr@gmail.com

\section{Antonio Lucas Nunes de Oliveira}

Formação acadêmica: médico generalista Instituição de atuação atual: Hospital UNIMED

Endereço completo: rua Costa Gomes, 208, Setor Central - CEP: 75901-050, Rio Verde - Goiás.

E-mail: amantoniolno@gmail.com

\section{Isadora Araújo Cabral}

Formação acadêmica: médica generalista

Instituição de atuação atual: Hospital Municipal Universitário, Rio Verde - Goiás Endereço completo: av. Jerônimo Martins, s/n, Parque Bandeirante - Rio Verde, Goiás

E-mail: isadora.araujocabral@gmail.com

\section{Guilherme Souza de Faria}

Formação acadêmica: médico cirurgião geral, residente em cirurgia da cabeça e pescoço

Instituição de atuação atual: Hospital Heliópolis

Endereço completo: rua Cônego Xavier, número 276, Sacomã, São Paulo - São Paulo

E-mail: amguilhermesf@gmail.com

\section{Jair Pereira de Melo Júnior}

Formação Acadêmica: físico, biólogo, professor doutor

Instituição de atuação atual: Universidade de Rio Verde

Endereço: Fazenda Fontes do Saber, Caixa Postal 104 - CEP: 75.901-970, Rio Verde - Goiás

E-mail: jnfjjunior@gmail.com

\section{Vicente Guerra Filho}

Formação Acadêmica: médico cirurgião do aparelho digestivo, professor doutor Instituição de atuação atual: Hospital Santa Terezinha

Endereço: Rua Agenor Diamantino, no 223, Pavimento 1, Vila Amália - CEP: 75.906-260, Rio

Verde, GO

E-mail: vicenteguerrafilho@yahoo.com.br 


\title{
RESUMO
}

o objetivo foi relatar um caso de megaesôfago idiopático que foi tratado por cardiomiotomia videolaparoscópica a Heller-Pinotti em um paciente masculino, de 29 anos. Foi realizada triagem inicial com anamnese e exame físico e o paciente alegou disfagia progressiva sem alterações dos hábitos intestinais associadas; exame físico abdominal sem alterações. A investigação diagnóstica laboratorial incluiu tireoideopatia, doença de Chagas e doença do refluxo gastroesofágico. Após extensa investigação foi detectada acalasia (confirmada por esofagomanometria) idiopática, sendo assim realizada a cardiomiotomia videolaparoscópica a Heller-Pinotti. Posteriormente ao tratamento cirúrgico, o paciente apresentou boa evolução pós-operatória, não havendo queixas de pirose ou regurgitação e desaparecendo a queixa de disfagia. Declara-se boa experiência com o tratamento cirúrgico proposto, no entanto, novos estudos se tornam necessários para aprimoramento da técnica cirúrgica e consequente maior benefício para o paciente com acalasia idiopática.

Palavras-chave: megaesôfago idiopático, acalasia idiopática, cardiomiotomia videolaparoscópica a Heller-Pinotti.

\begin{abstract}
the goal was to report a case of idiopathic achalasia that was treated by Heller-Pinotti's videolaparoscopic cardiomyotomy in a male patient of 29 years. The initial assessment was done with clinical history and physical examination and the patient claimed progressive dysphagia without changes in bowel habits associated; abdominal physical examination without abnormalities. Laboratory diagnostic investigation included thyroidopathy, Chagas' disease and gastroesophageal reflux disease. After extensive research, it was detected idiopathic achalasia (confirmed by esophageal manometry) and Heller- Pinotti's videolaparoscopic cardiomyotomy was realized. After surgery, the patient presented good postoperative evolution, with no complaints of heartburn or regurgitation and the dysphagia complaint had gone. We can declare good experience with the proposed surgical treatment, however, further studies are needed to make improvements in surgical technique and consequent greater benefit to the patient with idiopathic achalasia.
\end{abstract}

Keywords: idiopathic megaesophagus, idiopathic achalasia, videolaparoscopic Heller-Pinotti's cardiomyotomy.

\section{INTRODUÇÃO}

O megaesôfago é uma entidade caracterizada, primariamente, pela dilatação da luz esofágica. Dentre as condições que podem levar a esse quadro, destacam-se a doença de Chagas em sua forma crônica e quando não há causa descoberta (megaesôfago idiopático), ponto central deste trabalho (Nascimento et al., 2013) (Djeddi et al., 2011).

Atualmente, sabe-se que um dos prováveis mecanismos envolvidos na fisiopatologia do megaesôfago idiopático é a perda funcional de células ganglionares do plexo mioentérico do esôfago, o que determina aperistalse, principalmente do esôfago distal, hipertonia do esfíncter esofágico inferior (E.E.I.) e relaxamento incompleto do E.E.I. após deglutições - acalasia. Isso resulta em dificuldade de passagem do bolo alimentar do esôfago para o estômago (Pandolfino; Kahrilas, 2013) (Djeddi et al., 2011). 
As principais manifestações clínicas decorrentes dessa patologia incluem disfagia, regurgitação, soluços, halitose, perda de peso, pneumonia por aspiração, pirose e dor torácica (comum nas fases iniciais da acalasia). Os sintomas comumente se manifestam na faixa etária de 25 a 60 anos (Kahrilas; HIRANO, 2011) podendo determinar deficiências nutricionais e perda da qualidade de vida.

Para o diagnóstico de acalasia são utilizados, principalmente, exames contrastados como as radiografias da deglutição de bário e a manometria esofágica. A endoscopia digestiva alta desempenha papel importante, uma vez que auxilia na exclusão de diagnósticos diferenciais de disfagia como a pseudoacalasia por carcinoma do fundo gástrico ou do esôfago distal (Kahrilas; Hirano, 2011).

Dentre as opções terapêuticas disponíveis, pode-se mencionar o tratamento farmacológico com nitratos ou bloqueadores dos canais de cálcio; injeção de toxina botulínica; dilatação pneumática e miotomia a Heller com fundoplicatura parcial. O tratamento objetiva reduzir a pressão do E.E.I. para que a gravidade promova o esvaziamento gástrico. No entanto, o peristaltismo esofágico normal não é restaurado com a terapia (Kahrilas; Pandolfino, 2014).

\section{MATERIAL E MÉTODOS}

O paciente permitiu este relato por meio de termo de consentimento livre e esclarecido assinado e com cópia. $\mathrm{O}$ paciente em questão foi submetido a triagem inicial com anamnese e exame físico. A partir de suas queixas, foram solicitados exames complementares, dentre eles: glicemia de jejum; quimioluminescência para TSH (hormônio tireoestimulante) ultrassensível e para T4 (tiroxina) livre; hemograma completo; esofagogastroduodenoscopia; monitorização de pH esofágico de 24 horas; esofagomanometria; radiografia de tórax; radiografia de esôfago, estômago e duodeno após ingestão de bário; proteínas totais e frações; tempo de protrombina (T.A.P.); tempo de tromboplastina parcial ativada (TTPA); dosagem de creatinina e ureia séricas; dosagem de alanina aminotransferase (ALT) e aspartato aminotransferase (AST) séricas e eletrocardiograma de 12 derivações.

Para a exclusão do megaesôfago chagásico foram solicitadas três imunofluorescências indiretas para Doença de Chagas em ocasiões distintas. Uma vez diagnosticado o quadro de megaesôfago com acalasia idiopática, o paciente foi submetido à cardiomiotomia videolaparoscópica a Heller-Pinotti e, posteriormente, a endoscopia digestiva alta para averiguação do resultado anatômico da cirurgia realizada. 


\section{RESULTADOS E DISCUSSÃO}

Paciente do sexo masculino, 29 anos, possuía queixas de disfagia progressiva a sólidos há mais de 4 anos, sendo que no momento da consulta, a alimentação prosseguia apenas com líquidos. Negou alteração dos hábitos intestinais. Não possuía queixas urinárias. Negou presença palpitações.

Negou hábito de tabagismo; entretanto alegou etilismo social. Negou passado de cirurgias, hipertensão arterial, diabetes mellitus e uso de medicamentos. No entanto, o paciente apresentou pressão arterial de 145 x 100 mmHg. Não apresentou alterações à ectoscopia nem ao exame físico da cabeça (olhos, orelhas, nariz e garganta), do aparelho respiratório e do aparelho cardiovascular. O exame físico abdominal mostrou abdome plano, sem alterações à inspeção, com ruídos hidroaéreos presentes; à percussão: predominância de sons timpânicos; à palpação: flácido, indolor, fígado não palpável.

Quanto aos exames complementares, a glicemia de jejum $(92 \mathrm{mg} / \mathrm{dL})$; o TSH $(1,228 \mathrm{uUL} / \mathrm{mL})$ e a tiroxina livre $(1,17 \mathrm{ng} / \mathrm{dL})$; hemograma completo; a monitorização de $\mathrm{pH}$ esofágico de 24 horas; a radiografia de tórax; o T.A.P. (11 segundos com resultado de atividade de $100 \%$ ); o T.T.P.A. (30 segundos); as dosagens de creatinina $(0,90 \mathrm{mg} / \mathrm{dL})$ e ureia $(25,0 \mathrm{mg} / \mathrm{dL})$ séricas; as dosagens de ALT (17 U/L) e AST (16 U/L) séricas; e o eletrocardiograma de 12 derivações se revelaram dentro dos limites da normalidade. Houve discreta alteração de globulina sérica $(1,8 \mathrm{~g} / \mathrm{dL})$ e da relação albumina/globulina $(2,50)$ estando estas ligeiramente reduzidas; as proteínas totais $(6,3 \mathrm{~g} / \mathrm{dL})$ e a albumina sérica $(4,5 \mathrm{~g} / \mathrm{dL})$ estavam dentro da normalidade.

A esofagogastroduodenoscopia revelou luz esofágica de calibre aumentado e alargamento discreto dos pilares diafragmáticos sugerindo megaesôfago; o padrão vascular de esôfago distal se apresentou preservado e não houve anormalidades gástricas. O teste da urease para Helycobacter pilori foi negativo.

A esofagomanometria revelou relaxamento incompleto do esfíncter esofágico inferior às deglutições com percentual de relaxamento de $69 \%$ (VN >90\%) e pressão residual de $9 \mathrm{mmHg}(\mathrm{VN}<$ $8 \mathrm{mmHg}$ ). Revelou também complexos aperistálticos, isobáricos e de baixa amplitude no corpo do esôfago em resposta a todas as deglutições com água. O estudo manométrico foi compatível com megaesôfago avançado. A radiografia de esôfago, estômago e duodeno após ingestão de bário demonstrou megaesôfago grau III. 
Figura 1: radiografia de esôfago, estômago e duodeno após ingestão de bário demonstrando acalásia.

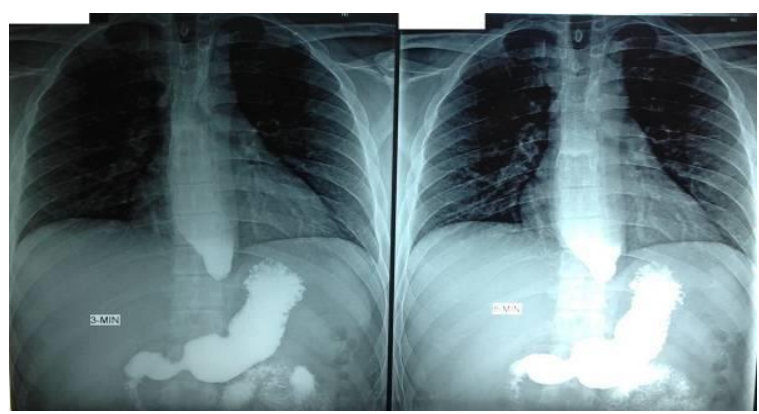

Todos os testes laboratoriais realizados para detecção da forma crônica da Doença de Chagas se mostraram não reagentes. Após a conclusão diagnóstica (megaesôfago idiopático), o paciente foi submetido à cardiomiotomia videolaparoscópica a Heller-Pinotti. Foi realizada a secção da membrana frenoesofágica até a identificação dos pilares diafragmáticos bilateralmente; isolou-se o esôfago distal e realizou-se a secção da musculatura longitudinal e circular nessa área esofágica a $6 \mathrm{~cm}$ e $3 \mathrm{~cm}$ baixo da transição esofagogástrica ficando a mucosa totalmente herniada. Posteriormente, foi realizada a fundoplicatura a Pinotti, sendo feitas três linhas de sutura: uma posterior, a segunda do estômago com a margem esquerda da miotomia e a terceira da parede anterior do estômago com a margem direita da miotomia. Não houve complicações.

Figura 2: cardiomiotomia videolaparoscópica a Heller-Pinoti.

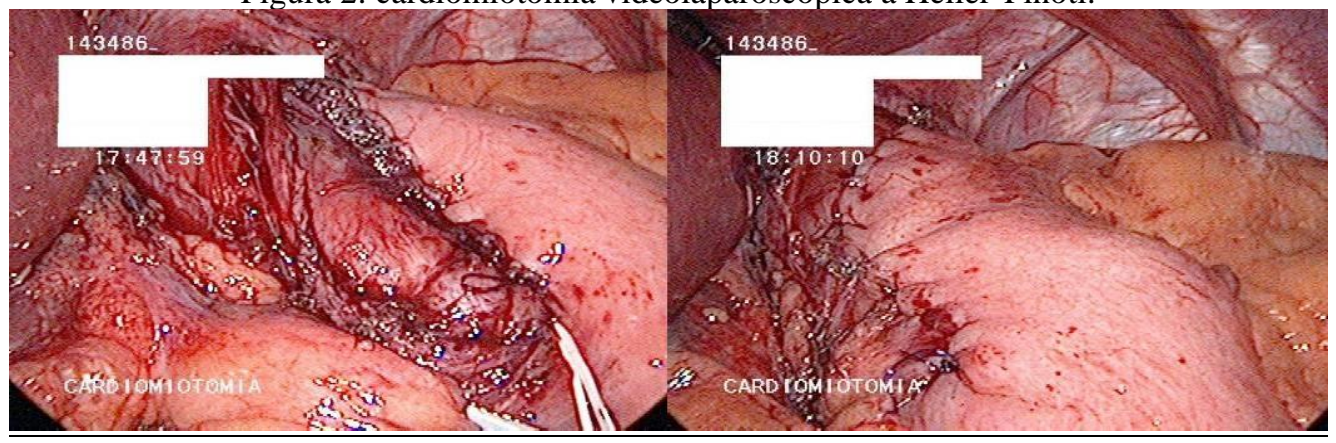


Recentemente, o paciente foi submetido a uma endoscopia digestiva alta que revelou passagem pelo cárdia sem qualquer resistência com evidências de válvula antirrefluxo competente.

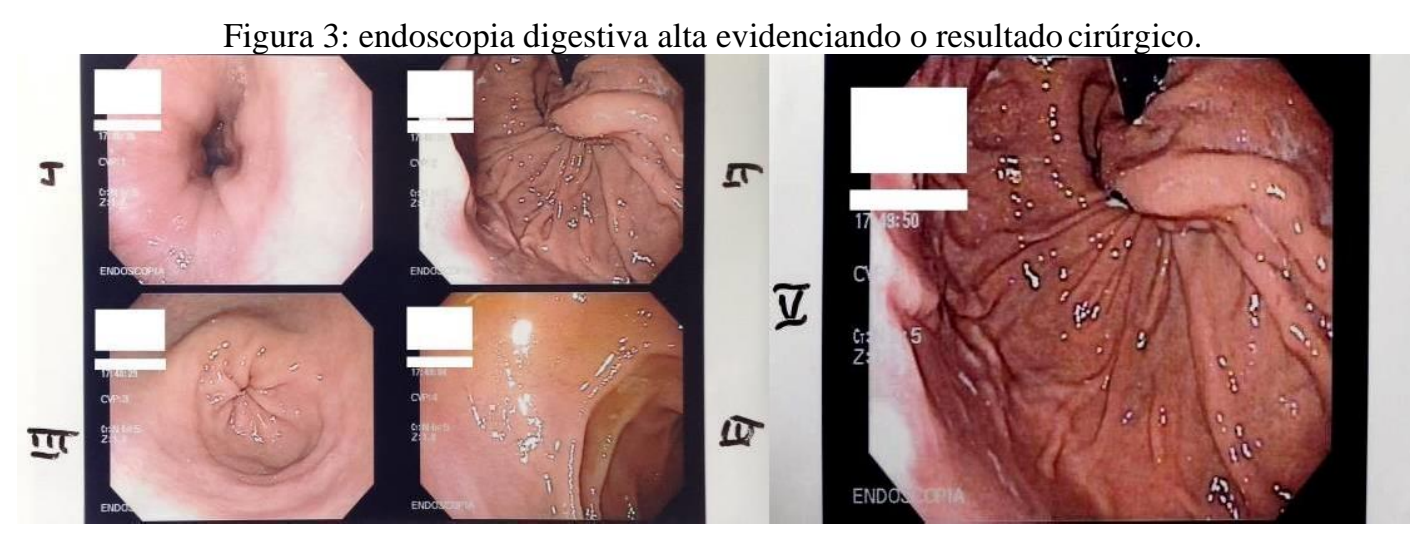

No $8^{\circ}$ dia de pós-operatório, o paciente evoluiu sem queixas de dor e com boa aceitação da dieta líquida completa; o exame físico se mostrou sem alterações e as feridas operatórias estavam em bom aspecto. A dieta foi evoluída para semipastosa.

Nos dias subsequentes o paciente continuou com boa evolução; apresentou perda ponderal de mais de $8 \mathrm{~kg}$, porém com ótima aceitação da dieta. Não houve queixas de disfagia, pirose ou regurgitação.

Diante de um quadro de disfagia, dor torácica e/ou pirose, é importante considerar a possibilidade de um distúrbio de motilidade esofágica. Segundo Kahrilas e Pandolfino (2014), as principais afecções de origem motora esofágica que cursam com essas manifestações incluem acalasia, espasmo esofágico distal (E.E.D.) e doença do refluxo gastroesofágico (DRGE) tornando uma boa investigação diagnóstica imprescindível para a realização de uma adequada conduta médica.

Metodologias envolvidas no diagnóstico e investigação da D.R.G.E. incluem a endoscopia digestiva alta (E.D.A.) e a monitorização do pH intraesofágico (Badillo; Francis, 2014). O paciente do estudo foi submetido a ambos os procedimentos. A E.D.A. não demonstrou sinais de esofagite estando a mucosa esofágica de aspecto normal. A monitorização do pH intraesofágico não registrou episódios de refluxo gastroesofágico. Além disso, o paciente não apresentou sintomas sugestivos de D.R.G.E., como pirose, regurgitação, sintomas atípicos ou extraesofágicos. Dessa forma, pode-se excluir a D.R.G.E. como possível etiologia do quadro.

De acordo com Kahrilas e Hirano (2011), o E.E.D. é diagnosticado por esofagomanometria e um dos achados clássicos presentes nesse exame é o relaxamento normal do E.E.I. durante a 
deglutição. A esofagomanometria a que foi submetido o paciente demonstrou hipertonia e relaxamento incompleto do

E.E.I. durante as deglutições. Assim, essa moléstia pode ser descartada como causadora das manifestações clínicas do paciente.

Outra condição que pode causar disfunção da peristalse esofágica determinando quadro de disfagia é o hipotireoidismo (Daher et. al, 2009). Para a exclusão de dismotilidade esofágica secundária a hipotireoidismo, foram realizadas quimioluminescências para TSH ultrassensível e para T4 livre, sendo os achados encontrados dentro do limite da normalidade.

A acalasia se tornou a principal hipótese diagnóstica. Os exames realizados (radiografias de deglutição de bário e manometria esofágica), conforme mostram os resultados supracitados sustentaram tal hipótese, confirmando a suspeita de acalasia idiopática.

Conforme Kahrilas e Pandolfino (2014), as outras doenças cujas consequências funcionais se assemelham às da acalasia idiopática são pseudoacalasia associada a malignidade ou doenças infiltrativas e a Doença de Chagas. A E.D.A. com achados normais auxiliou na exclusão de pseudoacalasia, além do fato de o paciente ser jovem (29 anos) e não ter fatores de risco significativos para doença maligna. Os três testes de imunfluorescência indireta realizados em momentos distintos não se mostraram reagentes, excluindo, assim, megaesôfago chagásico.

Através de todas as considerações investigativas explícitas acima, foi possível chegar ao diagnóstico do paciente e a próxima etapa que foi colocada em pauta foi o tratamento de sua condição.

De acordo com a American Gastroenterological Associaton, a miotomia cirúrgica e a dilatação pneumática graduada são a primeira escolha para o tratamento da acalasia em pacientes sem contraindicação (Yaghoobi et al., 2013). Os outros métodos, como a injeção de toxina botulínica e o tratamento farmacológico com nitratos ou bloqueadores dos canais de cálcio não possuem efeito marcante a longo prazo, o que exige uma terapêutica mais definitiva para que a qualidade de vida do paciente seja restaurada ao máximo possível.

Segundo Kahrilas e Pandolfino (2014), o apelo da miotomia é que esta oferece um método mais previsível de reduzir a pressão do E.E.I. do que a dilatação pneumática. Além disso, Yaghoobi et al. (2013) alega que muitos pacientes precisam de procedimentos repetidos de dilatação pneumática para alcançar taxas de resposta de 70 a $80 \%$, afora o fato de que o procedimento pode aumentar o risco de desenvolvimento de refluxo gastroesofágico.

Várias vantagens acompanham a cardiomiotomia videolaparoscópica a Heller, dentre elas, pode-se destacar a menor necessidade de procedimentos repetidos e os melhores resultados quanto à resolução dos sintomas com uma taxa de resposta de $80 \%$ a longo prazo. No entanto, uma desvantagem que pode acompanhar a miotomia a Heller é a possibilidade de desenvolvimento de 
refluxo gastroesofágico (Yaghoobi et al. 2013). Com vistas à minimização dessa desvantagem, associa-se a fundoplicatura a Pinotti a esse procedimento.

Com a análise cuidadosa do caso e dos métodos terapêuticos disponíveis, optou-se pela Cardiomiotomia laparoscópica a Heller-Pinotti. Houve excelente resposta terapêutica, sendo que no $25^{\circ}$ dia de pós-operatório, o paciente alegou que não havia disfagia, pirose ou regurgitação.

\section{CONCLUSÃO}

A cardiomiotomia videolaparoscópica a Heller-Pinotti se mostra um método terapêutico promissor no tratamento da acalasia. Esse procedimento foi introduzido em 1991 e, desde então, tem sua popularidade apenas aumentando.

Relata-se boa experiência com tal abordagem. Novos estudos se fazem necessários para que a técnica operatória seja cada vez mais aprimorada e refinada, minimizando o risco de qualquer evento adverso. Dessa forma, o paciente estará sendo melhor beneficiado e atendido quanto às suas necessidades. 


\section{REFERÊNCIAS BIBLIOGRÁFICAS}

BADILLO, Raul; FRANCIS, Dawn. Diagnosis and treatment of gastroesophageal reflux disease. World Journal Of Gastrointestinal Pharmacology And Therapeutics, v. 5, n. 3, p.105-112, 6 ago. 2014.

DAHER, Ronald et al. Consequences of dysthyroidism on the digestive tract and viscera. World Journal Of Gastroenterology, v. 15, n. 23, p.2834-2838, 21 jun. 2009.

DJEDDI, D. et al. Another case of idiopathic megaesophagus in a girl with growth hormone deficiency. Clinics And Research In Hepatology And Gastroenterology, v. 35, n. 11, p.768-770, nov. 2011.

KAHRILAS, Peter J.; HIRANO, Ikuo. Doenças do Esôfago. In: LONGO, Dan L. et al (Org.). Medicina Interna de Harrison. 18. ed. Porto Alegre: AMGH Editora Ltda., 2013. Cap. 292. p. $2427-$ 2437.

KAHRILAS, Peter J.; PANDOLFINO, John E. Função Neuromuscular Esofágica e Transtornos da Motilidade. In: FELDMAN, Mark; FRIEDMAN Lawrence S.; BRANDT, Lawrence J. Sleisenger \& Fordtran Tratado Gastrointestinal e Doenças do Fígado: Fisiopatologia/Diagnóstico/Tratamento. 9. Ed. Rio de Janeiro: Elsevier, 2014. Cap. 42, p. 689-716.

NASCIMENTO, Rodolfo Duarte et al. An imbalance between substance P and vasoactive intestinal polypeptide might contribute to the immunopathology of megaesophagus after Trypanosoma cruzi infection. Human Pathology, v. 44, n. 2, p.269-276, fev. 2013.

PANDOLFINO, John E.; KAHRILAS, Peter J.. Presentation, Diagnosis, and Management of Achalasia. Clinical Gastroenterology And Hepatology, v. 11, n. 8, p.887-897, ago. 2013.

YAGHOOBI, Mohammad et al. Laparoscopic Heller's myotomy versus pneumatic dilation in the treatment of idiopathic achalasia: a meta-analysis of randomized, controlled trials. Clinical Endoscopy, v. 78, n. 3, p.468-475, set. 2013. 